\title{
Joule Heating and Viscous Dissipation on Effects on MHD Flow over a Stretching Porous Sheet Subjected to Power Law Heat Flux in Presence of Heat Source
}

\author{
Khaled K. Jaber \\ Department of Mathematics, Faculty of Science and Information Technology, Zarqa University, Zarqa, Jordan \\ Email: khaledjaber4@yahoo.com
}

How to cite this paper: Jaber, K.K. (2016) Joule Heating and Viscous Dissipation on Effects on MHD Flow over a Stretching Porous Sheet Subjected to Power Law Heat Flux in Presence of Heat Source. Open Journal of Fluid Dynamics, 6, 156-165. http://dx.doi.org/10.4236/oifd.2016.63013

Received: June 8, 2016

Accepted: September 10, 2016

Published: September 13, 2016

Copyright (c) 2016 by authors and Scientific Research Publishing Inc. This work is licensed under the Creative Commons Attribution International License (CC BY 4.0).

http://creativecommons.org/licenses/by/4.0/

\begin{abstract}
In the present work we investigate the effects of Joule heating and viscous dissipation on MHD fluid flow. The viscous incompressible fluid flows over a stretching porous horizontal sheet subjected to power law heat flux in presence of heat source. The equations of momentum and heat transfer governing the problem are transformed into a system of dimensionless differential equations, which in turn solved numerically using shooting technique. The effects of the Joule heating parameter, permeability parameter, heat source parameter, Eckert number and Prandtl number are discussed and tabulated.
\end{abstract}

\section{Keywords}

Viscous Dissipation, Heat Transfer, Porous Medium, Heat Flux

\section{Introduction}

Studies of boundary layer flow of viscous fluid and heat transfer on a continuously moving surface are of great importance in view of their relevance to many technological processes, the manufacturing of aerodynamic extrusion of plastic sheets, cooling of metallic paths rapidly, many industries like fiber, glass and polymers. Sakiadis [1] was the first researcher who studied the boundary layer flow of a viscous fluid adjacent to a continuously stretching vertical sheet. A combined experimental and analytically study of the laminar and turbulent flow and temperature distribution in the boundary layer on a continuously moving surface has been carried out by Tsou et al. [2]. Erickson et al. [3] and Gupta et al. [4] extended Sakiadis problem, they taking into account suction or 
blowing of the fluid at the moving surface. Since that many researcher extended the problem in various ways. Afzal et al. [5] and Banks [6] considered the power law stretching of the moving sheet. Magnetohydrodynamics (MHD) is the study of the magnetic properties of electrically conducting fluids, such as plasmas, liquid metals, and salt water or electrolytes. Hannes Alfven [7] was the first who studying MHD, He described the class of MHD waves now known as Alfvén waves, for which he received the Nobel Prize in Physics in 1970. Recently, magneto hydrodynamic flow and heat transfer have been studied by many researchers such as Abo-Eldahab [8] and Chakarabati et al. [9]. Due to its ever increasing engineering applications such as the flow through packed beds, environmental pollution, blood theology and many other applications the extension of the problem to porous sheet attracted many researchers. Varjravelu et al. [10] studied the laminar flow and heat transfer of a viscous fluid over a stretching surface with viscous dissipation. Chamkha [11] studied the problem of thermal radiation effects on MHD forced convection laminar flow adjacent to a non-isothermal wedge in the presence of a heat source or sink. Abo-Eldahab et al. [12] studied the viscous dissipation and Joule heating effects on magneto hydrodynamic free convection from a vertical plate with power-law variation in surface temperature in the presence of Hall and ion-slip currents. Jaber [13] studied the Transient magneto hydrodynamic mixed double diffusive convection along a vertical plate embedded in a non-Darcy porous medium with suction or injection. Abo-Eldahab and Elbarbary [14] examined heat and mass transfer over a vertical plate in the presence of a uniform and strong magnetic field and Hall current. Abo-Eldahab and El Aziz [15] investigated the effects of Hall current and Joule heating on electrically conducting fluid past a semi-infinite plate with strong magnetic field and heat generation/absorption. Jaber [16] studied the effect of Hall currents, and variable viscosity on free convective flow past a semi-infinite continuously stretching plate in presence of radiation. Jaber [17] studied the effect of Hall currents and variable fluid properties on magneto hydrodynamics flow past a continuously stretching vertical plate in the presence of radiation. Viscous dissipation effect plays an important role in natural convection in various devices which are subjected to large variations of gravitational force or which operate at high speeds Jaber [18] examined the effects of viscous dissipation and joule heating on magneto hydrodynamics flow of a fluid with variable properties past a stretching vertical plate. Mandal, et al. [19] studied the effect of Hall current on MHD couette flow between thick arbitrarily conducting Plates in a rotating system. Heat flux or thermal flux is the rate of heat energy transfer through a given surface per unit time Mandal et al. [20] study the boundary layer flow and heat transfer towards an exponentially stretching porous sheet embedded in a porous medium with variable surface heat flux.

The purpose of this present work is to study the combined effects of Joule heating and viscous dissipation on laminar flow and heat transfer of a viscous fluid over a horizontal porous sheet subjected to power law heat flux in presence of heat source, also the plate is continuously stretching. The governing equations are transformed into dimensionless ordinary differential equations. Numerical calculations up to desired level 
of accuracy were carried out to investigate the effects of all parameters involved in the problem under consideration to illustrate the results graphically.

\section{Mathematical Formulation}

The present work generalizes the problem of heat transfer over a stretching porous sheet subjected to power law heat flux in presence of heat source which presented by Hitesh Kumar [21]. Consider the laminar steady two dimensional flow of an incompressible viscous fluid over a horizontal plate coinciding with the plane $y=0$. A uniform strong magnetic field of strength $B_{0}$ is subjected normal to the plate. The plate is subjected to a power law heat flux in presence of heat source. The plate is continuously stretching by two equal powers with opposite directions. The speed is assumed to be proportional to its distance from the slit point. The flow is considered to be in the $\mathrm{x}$-direction which is taken along the plate, see Figure 1.

Under these assumptions and taking into account the Boussinesq approximation, the boundary layer laminar flow is governed by the following equations:

$$
\begin{aligned}
& \frac{\partial u}{\partial x}+\frac{\partial v}{\partial x}=0 \\
& u \frac{\partial u}{\partial x}+v \frac{\partial u}{\partial y}=v \frac{\partial^{2} u}{\partial y^{2}}-\frac{\sigma B_{0}^{2}}{\rho} u \\
& u \frac{\partial T}{\partial x}+v \frac{\partial T}{\partial y}=\frac{k}{\rho C_{P}} \frac{\partial^{2} T}{\partial y^{2}}-\frac{v}{C_{P}}\left(\frac{\partial u}{\partial y}\right)^{2}+\frac{\sigma B_{0}^{2}}{\rho C_{P}} u^{2}
\end{aligned}
$$

The physical problem suggested the following initial and boundary conditions:

$$
\begin{aligned}
& \text { at } y=0: u=c x, v=-v_{0}, \frac{\partial T}{\partial y}=B x^{2} \\
& \text { at } y \rightarrow \infty: u=v=0, T=T
\end{aligned}
$$

where $c$ is the stretching rate which considered to be a positive integer.

The continuity equation suggested the solution

$$
u=c x f^{\prime}(\eta)_{\infty}, \quad v=-\sqrt{v c} f(\eta), \quad \eta=\sqrt{c v^{-1}} y, \quad T=T_{\infty}+B x^{2} \sqrt{v c^{-1}} \theta(\eta)
$$

Using these dimensionless parameters the governing equations, Equations (2) and (3) can be reduced to the following system of ordinary differential equations

$$
\begin{aligned}
& f^{\prime \prime \prime}+f f^{\prime \prime}-f^{\prime 2}-M f^{\prime}=0 \\
& \theta^{\prime \prime}+\operatorname{Pr} f \theta^{\prime}-2 \operatorname{Pr} f^{\prime} \theta-\beta \theta+\operatorname{PrEc} f^{\prime 2}+E c J f^{\prime 2}=0
\end{aligned}
$$

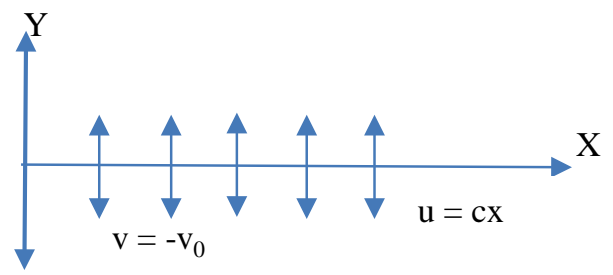

Figure 1. Physical coordinate system. 
The boundary conditions are transformed to:

At the surface of the plate $f^{\prime}(0)=1, f(0)=\frac{v_{0}}{\sqrt{c \eta}}, \theta^{\prime}(0)=1$

Also, as $\eta \rightarrow \infty: f^{\prime}=f=\theta=0$

where $\operatorname{Pr}=\frac{\rho v C_{P}}{k}$ is the Prandtl number, $E c=\frac{c^{\frac{5}{2}}}{B C_{P} \sqrt{v}}$ is the Eckert number $J=\frac{\sigma B_{0}^{2} v C_{P}}{k}$ is the Joule heating parameter, $\beta=\frac{v Q}{c k}$ is the heat source parameter.

The physical quantities of interest Nusselt number and the local shear stress $\tau_{w}$ are defined as

$$
q_{w}=-k\left(\frac{\partial T}{\partial y}\right)_{y=0}
$$

Thus the Nusselt number is given by

$$
N u=\frac{q_{w} x}{k}=-B x^{3} \theta^{\prime}(0)
$$

The wall shear stress is defined as

$$
\tau_{w}=\mu\left(\frac{\partial u}{\partial y}\right)_{y=0}=\frac{\mu x c^{\frac{3}{2}}}{\sqrt{v}} f^{\prime \prime}(0)
$$

The numerical values of $f^{\prime}(0)$ and $\theta^{\prime}(0)$ for several values of the Magnetic number, Joule heating parameter, heat source parameter, Eckert number and Prandtl number are tabulated in Table 1.

Table 1. Variation of the dimensionless skin friction and local Nusselt number for various parameters.

\begin{tabular}{ccccccc}
\hline $\boldsymbol{P r}$ & $E \boldsymbol{c}$ & $\boldsymbol{M}$ & $J$ & $\beta$ & $-\boldsymbol{f}(0)$ & $\boldsymbol{\theta}(0)$ \\
\hline 0.72 & 3 & 1 & 1 & 1 & 2.00007 & 0.719796 \\
2 & 3 & 1 & 1 & 1 & 2.00007 & 1.26137 \\
5 & 3 & 1 & 1 & 1 & 2.00007 & 1.58979 \\
0.72 & 5 & 1 & 1 & 1 & 2.00007 & 1.55876 \\
0.72 & 8 & 1 & 1 & 1 & 2.00007 & 2.8172 \\
0.72 & 3 & 3 & 1 & 1 & 2.56155 & 1.00835 \\
0.72 & 3 & 5 & 1 & 1 & 3 & 1.25537 \\
0.72 & 3 & 1 & 3 & 1 & 2.00007 & 1.36839 \\
0.72 & 3 & 1 & 5 & 1 & 2.00007 & 2.01699 \\
0.72 & 3 & 1 & 1 & 3 & 2.00007 & 0.437762 \\
0.72 & 3 & 1 & 1 & 5 & 2.00007 & 0.315727 \\
\hline
\end{tabular}




\section{Numerical Technique}

The transformed governing system consists of two non-linear ordinary differential equations. They were solved numerically up to desired level of accuracy using the fourth order Runge-Kutta method with the shooting technique. The effect of all the involved parameters on the velocity and temperature profiles, the local Nusselt number and the shear stress is presented and discussed. The effects of the involved parameters were presented graphically as shown in Figures 2-7. Figure 2 and Figure 3 present the effect of the magnetic parameter $M$ on the velocity and the temperature profiles, the increasing of magnetic parameter $M$ causes decreasing in the velocity and give rise to increase the temperature profiles. Figure 4 shows that the increasing of the Eckert number leads to increase the temperature profiles. The effect of the Prandtl number is presented in Figure 5, it is obvious from the figure that the increasing in the Prandtl number leads to a markedly increasing in the initial magnitude of the temperature in the boundary

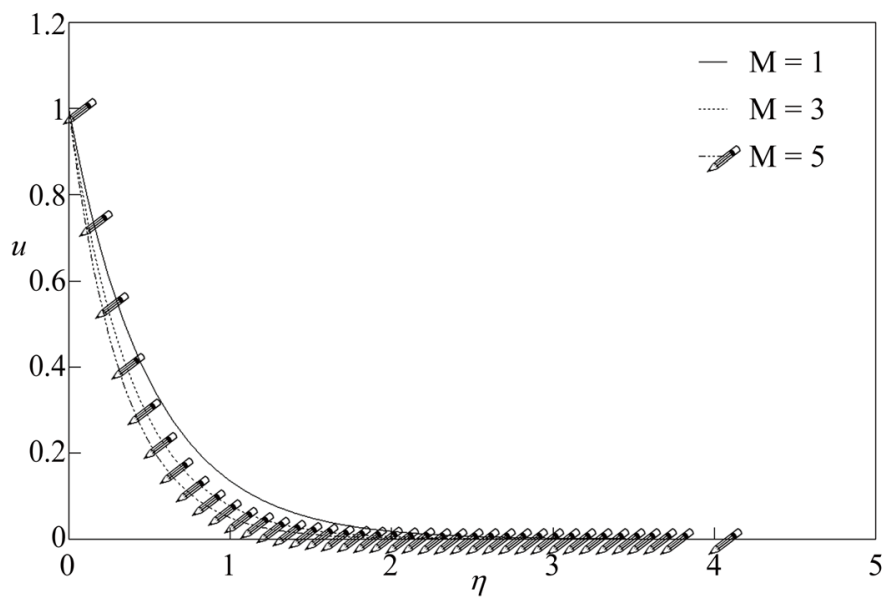

Figure 2. Variation of dimensionless velocity $f$ for various values of magnetic number $M$ with $P r=0.72, \beta=1, E c=3, J=3$.

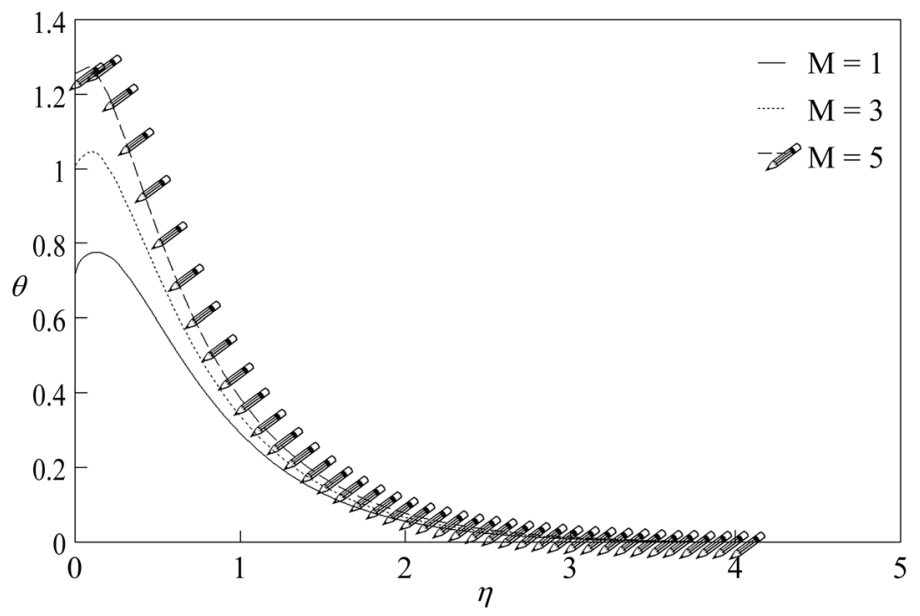

Figure 3. Variation of dimensionless temperature $\theta$ for various values of magnetic number $M$ with $P r=0.72, \beta=1, E c=3, J=3$. 


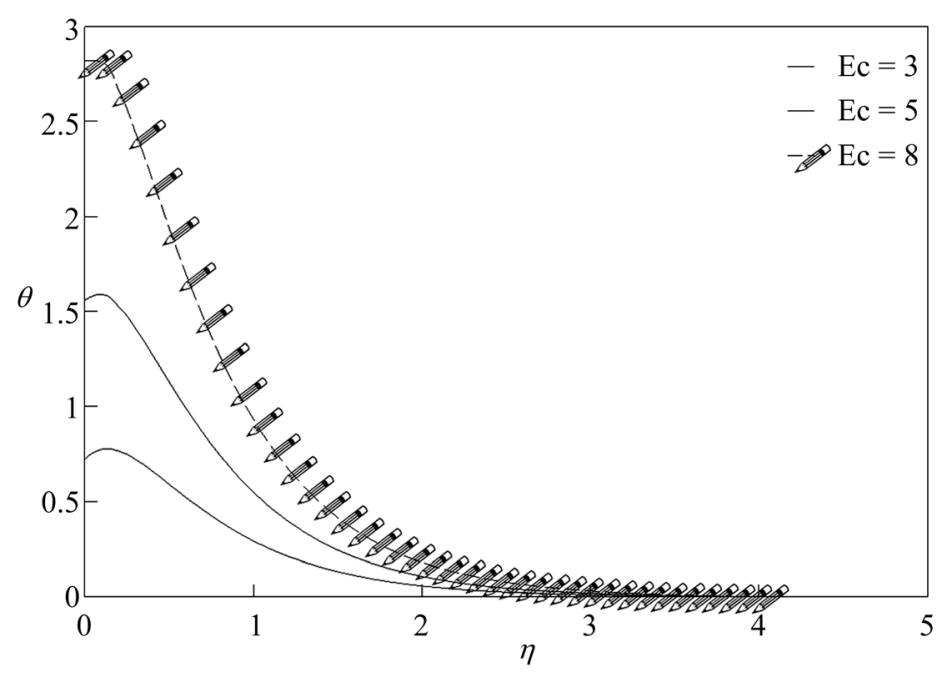

Figure 4. Variation of dimensionless temperature $\theta$ for various values of Eckert number $E c$ with $\operatorname{Pr}=0.72, \beta=1, J=3, M=1$.

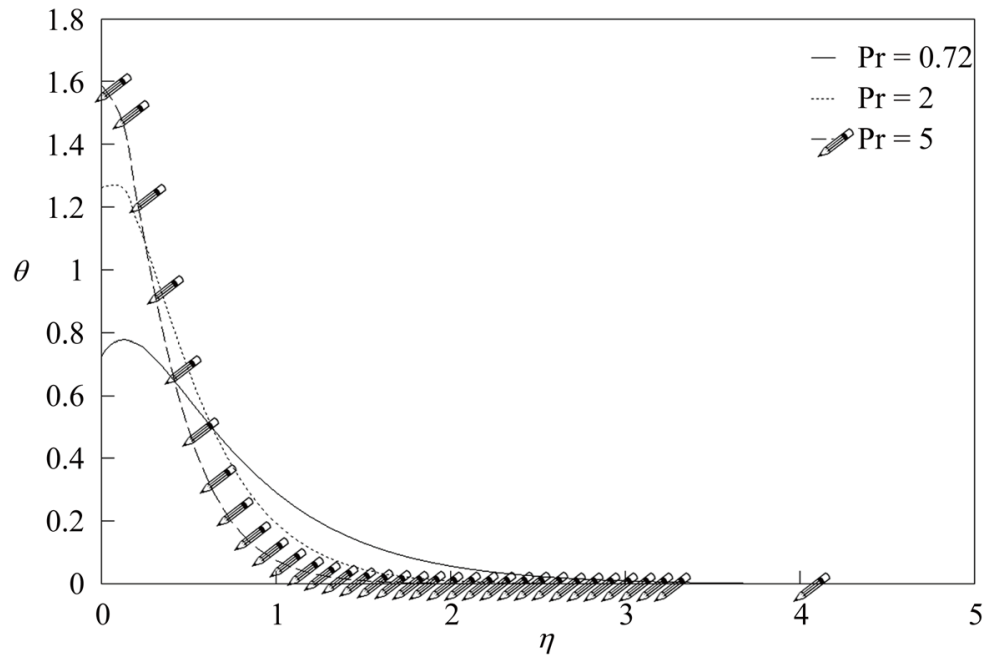

Figure 5. Variation of dimensionless temperature $\theta$ for various values of $P r$ and $t$ number $\operatorname{Pr}$ with $\beta=M=1, J=3, E c=3$.

layer closed to the plate while this effect takes the reverse order as the fluid goes far from the plate i.e. temperature profile decreases as the fluid get far away from the plate. Figure 6 investigates the effect of the Joule heating parameter, the increase in the Joule heating parameter causes markedly increase in the magnitude of the temperature in the boundary layer. Finally, Figure 7 presents the effect of the heat source parameter $\beta$ on temperature profile, it is clear that the increasing in the heat source parameter $\beta$ leads to decrease the temperature in the boundary layer. Table 1 shows that the local skin friction for the flow decreases as the magnetic parameter $M$ increases, while other parameters do not affect the local skin friction. Also, it is easily seen that the increasing in the values of $P r, E c, M$ and Joule heating parameters increase the local Nusselt number, while it is decreased by the increasing of the heat source parameter $\beta$. 


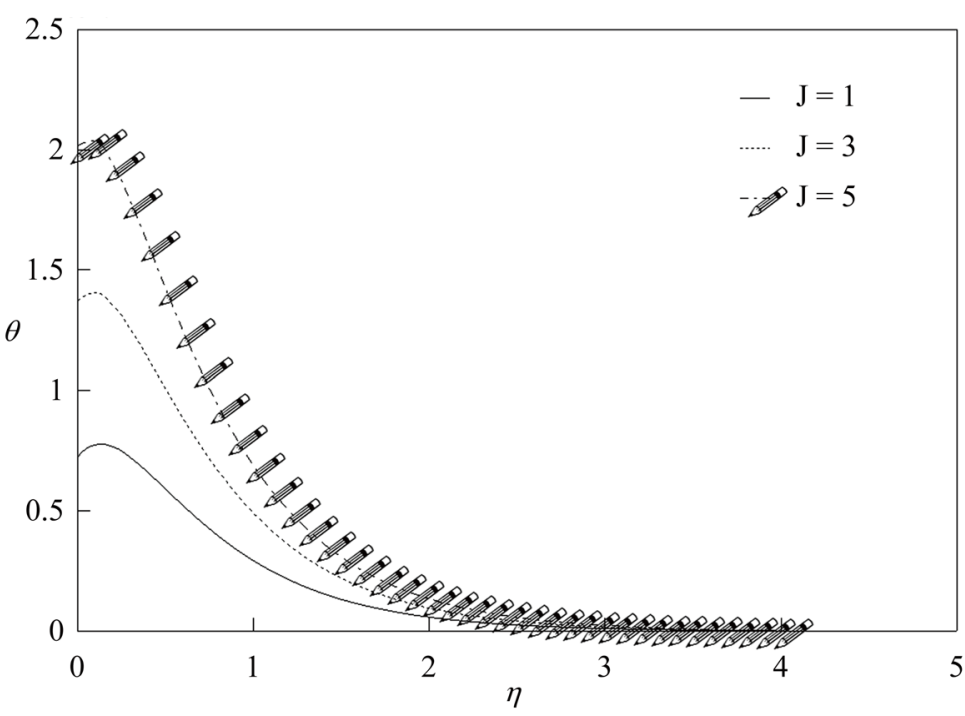

Figure 6. Variation of dimensionless temperature $\theta$ for various values of Joule heating parameter $J$ with $E \mathcal{C}=3, \operatorname{Pr}=0.72, \beta=M=1$.

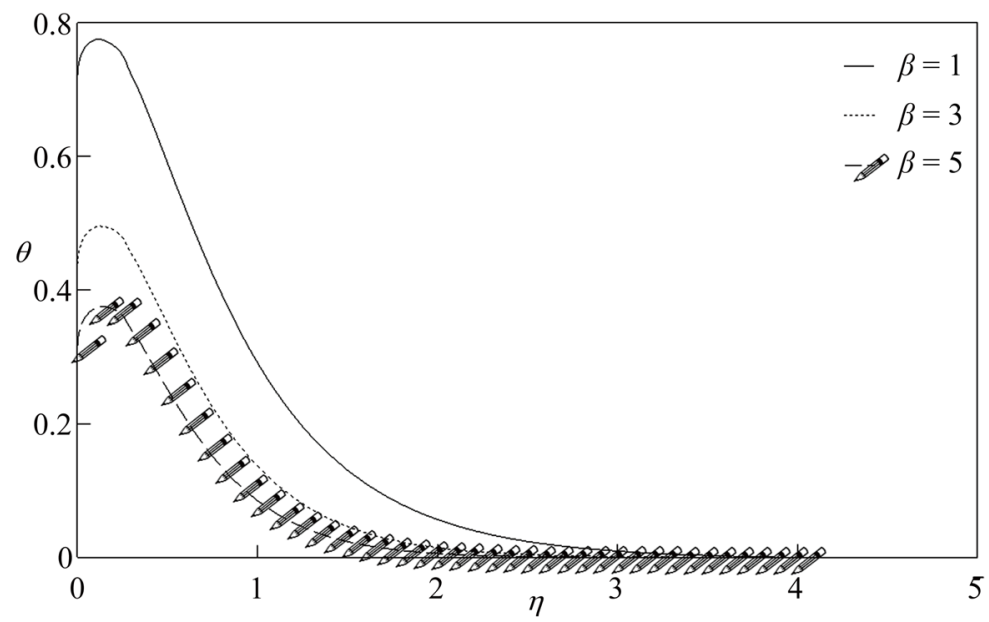

Figure 7. Variation of dimensionless temperature $\theta$ for various values of heat source parameter $\beta$ with $E \mathcal{C}=J=3, \operatorname{Pr}=0.72, M=1$.

\section{Conclusions and Remarks}

The combined effects of viscous dissipation and Joule heating on the steady, laminar, convection heat transfer over a horizontal plate is investigated, the plate is permeable and continuously stretching with a power-law heat source. The governing equations are transformed into a system of ordinary differential equations that solved numerically. Results are tabulated and presented graphically for various values of the involved parameters.

The present study shows that:

1) Increasing the magnetic parameter $M$ increases the temperature and the local Nusselt number and decreasing the velocity and the local skin friction.

2) Increasing the Eckert number increases both the local Nusselt number and tem- 
perature profiles.

3) Increasing the Prandtl number Pr increases both the initial values of temperature and the local Nusselt number.

4) As expected the increasing in the Joule heating parameter increases the temperature profile and local Nusselt number, also it does not affect the skin friction.

5) As the heat source parameter $\beta$ increases, the temperature and local skin friction decrease.

6) No effect of $J, E c, \operatorname{Pr}$ and heat source parameter $\beta$ on velocity profiles so no figures are presented herein to limit the figures number, also they do not affect the local skin friction.

\section{Acknowledgements}

This research is funded by the Deanship of Research and Graduate Studies in Zarqa University/Jordan.

\section{References}

[1] Sakiadis, B.C. (1961) Boundary Layer Behavior on Continuous Solid Surface. American Institute of Chemical Engineers Journal, 7, 26-28.

[2] Tsou, F.K., Sparrow, E.M. and Goldstein, R.J. (1967) Flow and Heat Transfer in the Boundary Layer on a Continuous Moving Surface. International Journal of Heat and Mass Transfer, 10, 219-235. http://dx.doi.org/10.1016/0017-9310(67)90100-7

[3] Erickson, L.E., Fan, L.T. and Fox, V.G. (1966) Heat and Mass Transfer of a Moving Continuous Flat Plate with Suction or Injection. Industrial \& Engineering Chemistry Fundamentals, 5, 19-25. http://dx.doi.org/10.1021/i160017a004

[4] Gupta, P.S. and Gupta, A.S. (1977) Heat and Mass Transfer on a Stretching Sheet with Suction or Blowing. The Canadian Journal of Chemical Engineering, 55, 744-746. http://dx.doi.org/10.1002/cjce.5450550619

[5] Afzal, N. and Varshney, I.S. (1980) The Cooling of low Heat Resistance Stretching Sheet Moving Through a Fluid. Heat and Mass Transfer, 14, 289-293.

[6] Banks, W.H.H. and Zaturska, M.B. (1986) Eigensolutions in Boundary-Layer Flow Adjacent to a Stretching Wall. Ima Journal of Applied Mathematics, 36, 263-273.

[7] Alfvén, H. (1942) Existence of Electromagnetic-Hydrodynamic Waves. Nature, 150, 405406.

[8] Abo-Eldahab, E.M. and Salem, A.M. (2004) Hall Effect on MHD Free Convection Flow of a Non-NewtonianPower Law Fluid at a Stretching Surface. International Communications in Heat and Mass Transfer, 31, 343-354. http://dx.doi.org/10.1016/j.icheatmasstransfer.2004.02.005

[9] Chakrabarti, A. and Gupta, A.S. (1979) Hydromagnetic Flow and Heat Transfer over a Stretching Sheet. Quarterly of Applied Mathematics, 37, 73-78.

[10] Vajravelu, K. and Hadjinicolaou, A. (1993) Heat Transfer in a Viscous Fluid over a Stretching Sheet with Viscous Dissipation and Internal Heat Generation. International Communications in Heat and Mass Transfer, 20, 417-430. http://dx.doi.org/10.1016/0735-1933(93)90026-R

[11] Chamkha, A.J., Mujtaba, M., Quadri, A. and Issa, C. (2003) Thermal Radiation Effects on MHD Forced Convection Flow Adjacent to a Non-Isothermal Wedge in the Presence of a 
Heat Source or Sink. Heat and Mass Transfer, 39, 305-312.

[12] Abo-Eldahab, E.M. and El Aziz, M.A. (2005) Viscous Dissipation and Joule Heating Effects on MHD-Free Convection from a Vertical Plate with Power-Law Variation in Surface Temperature in The presence of Hall and Ion-Slip Currents. Applied Mathematical Modelling, 29, 579-595. http://dx.doi.org/10.1016/j.apm.2004.10.005

[13] Jaber, K.K. (2012) Transient MHD Mixed Double Diffusive Convection along a Vertical plate Embedded in a Non-Darcy Porous Medium with Suction or Injection. Journal of Mathematics and Statistics, $8,1$.

[14] Aboeldahab, E.M. and Elbarbary, E.M.E. (2001) Hall Current Effect on Magnetohydrodynamic Free-Convection Flow past a Semiinfinite Vertical Plate with Mass Transfer. International Journal of Engineering Science, 39, 1641-1652.

http://dx.doi.org/10.1016/S0020-7225(01)00020-9

[15] Abo-Eldahab, E.M. and Mohamed, A.E. (2004) Hall Current and Ohmic Heating Effects on Mixed Convection Boundary Layer Flow of a Micropolar Fluid from a Rotating Cone with Power-Law Variation in Surface Temperature. International Communications in Heat and Mass Transfer, 31, 751-762.

[16] Jaber, K.K. (2014) Combined Effects of Hall Current and Variable Viscosity on NonNewtonian MHD Flow past a Stretching Vertical Plate. Journal of Advances in Mathematics, 7, 1358-1366.

[17] Jaber, K.K. (2014) Effect of Hall Currents and Variable Fluid Properties on MHD Flow past Stretching Vertical Plate by the Presence of Radiation. Journal of Applied Mathematics and Physics, 2, 888-902.

[18] Jaber, K.K. (2014) Effect of Viscous Dissipation and Joule Heating on MHD Flow of a Fluid with Variable Properties past a Stretching Vertical Plate. European Scientific Journal, 10.

[19] Mandal, G. and Mandal, K.K. (1983) Effect of Hall Current on MHD Couette Flow between Thick Arbitrarily Conducting Plates in a Rotating System. Journal of the Physical Society of Japan, 52, 470-477. http://dx.doi.org/10.1143/JPSJ.52.470

[20] Mandal, I.C. and Mukhopadhyay, S. (2013) Heat Transfer Analysis for Fluid Flow over an Exponentially Stretching Porous Sheet with Surface Heat Flux in Porous Medium. Ain Shams Engineering Journal, 4, 103-110. http://dx.doi.org/10.1016/j.asej.2012.06.004

[21] Kumar, H. (2011) Heat Transfer over a Stretching Porous Sheet Subjected to Power Law Heat Flux in Presence of Heat Source. Thermal Science, 15, S187-S194.

http://dx.doi.org/10.2298/TSCI100331074K 


\section{Nomenclature}

\begin{tabular}{cccc}
\hline$u$ & Velocity component in x-direction & $X$ & Stream wise coordinate \\
$V$ & Velocity component in y-direction & $y$ & Direction normal to the plate \\
$E c$ & Eckert number & & Greek Symbols \\
$P r$ & Prandtl number & $\beta$ & Heat source parameter \\
$f$ & Dimensionless stream function & $\eta$ & Pseudo similar variable \\
$C_{P}$ & Specific heat at constant pressure & $J$ & Joule heating parameter \\
$k$ & Thermal conductivity & $\mu$ & Dynamical viscosity \\
$N u$ & Nusselt number & $\rho$ & Density \\
$\tau$ & Shearing stress & $v$ & Kinematical viscosity \\
$T$ & Temperature & $\theta$ & Dimensionless temperature \\
$q_{W}$ & The local heat flux & & Subscripts \\
$c$ & The stretching rate & $W$ & Property at the wall \\
$K$ & Permeability of the porous medium & $\infty$ & Free stream condition \\
$J$ & Joule heating parameter & & Superscripts \\
$M$ & Magnetic number & & Differentiation with respect to $\eta$ only \\
\hline
\end{tabular}

Submit or recommend next manuscript to SCIRP and we will provide best service for you:

Accepting pre-submission inquiries through Email, Facebook, LinkedIn, Twitter, etc. A wide selection of journals (inclusive of 9 subjects, more than 200 journals)

Providing 24-hour high-quality service

User-friendly online submission system

Fair and swift peer-review system

Efficient typesetting and proofreading procedure

Display of the result of downloads and visits, as well as the number of cited articles Maximum dissemination of your research work

Submit your manuscript at: http://papersubmission.scirp.org/ 